\title{
Interaction and cross-resistance of cisplatin and pemetrexed in malignant pleural mesothelioma cell lines
}

\author{
MIYAKO KITAZONO-SAITOH ${ }^{1}$, YUICHI TAKIGUCHI ${ }^{2}$, SATORU KITAZONO ${ }^{1}$, \\ HIRONORI ASHINUMA ${ }^{1}$, ATSUSHI KITAMURA ${ }^{1}$, YUJI TADA ${ }^{1}$, KATSUSHI KUROSU ${ }^{1}$, \\ EMIKO SAKAIDA $^{2}$, IKUO SEKINE ${ }^{2}$, NOBUHIRO TANABE ${ }^{1}$, \\ MASATOSHI TAGAWA ${ }^{3}$ and KOICHIRO TATSUMI ${ }^{1}$ \\ Departments of ${ }^{1}$ Respirology and ${ }^{2}$ Medical Oncology, Graduate School of Medicine, Chiba University; \\ ${ }^{3}$ Division of Pathology and Cell Therapy, Chiba Cancer Center, Chiba, Japan
}

Received January 30, 2012; Accepted April 13, 2012

DOI: $10.3892 /$ or.2012.1799

\begin{abstract}
Although cisplatin and pemetrexed are key drugs in the treatment of malignant pleural mesothelioma, their drugdrug interactions, cross-resistance and resistance mechanisms in malignant pleural mesothelioma are not well understood. In the present study, the interaction of these 2 agents was determined by clonogenic assays followed by isobologram analysis of 4 human malignant pleural mesothelioma cell lines. The cell lines were exposed to the agents using a stepwise dose-escalation method to establish drug-resistant sublines. Thymidylate synthase mRNA expression was evaluated in the drug-resistant sublines. As a consequence, cisplatin and pemetrexed had synergistic effects in 3 cell lines and an additive effect in the fourth cell line. The former 3 cell lines showed similar pemetrexed sensitivity in the parental cells and their cisplatin-resistant sublines, whereas the fourth cell line exhibited cross-resistance. In contrast, cisplatin had diverse effects on pemetrexed-resistant sublines. High thymidylate synthase expression did not correlate with natural pemetrexed resistance. Elevated thymidylate synthase expression correlated with acquired pemetrexed resistance in 2 sublines. In conclusion, cisplatin and pemetrexed showed synergistic activity and no cross-resistance in 3 of the 4 malignant pleural mesothelioma cell lines, suggesting the clinical relevance of their combination in chemotherapy. Thymidylate synthase expression did not necessarily correlate with pemetrexed resistance. The information together with the experimental model presented here would be useful for further investigating therapeutic targets of malignant mesothelioma.
\end{abstract}

Correspondence to: Dr Yuichi Takiguchi, Department of Medical Oncology, Graduate School of Medicine, Chiba University, 1-8-1, Inohana, Chuo-ku, Chiba 260-8670, Japan

E-mail: takiguchi@faculty.chiba-u.jp

Key words: mesothelioma, cisplatin, pemetrexed, cross-resistance, synergism

\section{Introduction}

Malignant pleural mesothelioma (MPM), often linked to asbestos exposure, is one of the most lethal cancers with a rapidly increasing incidence. For example, the latest available data indicate that the incidence of MPM in Australia is 40 cases per million and is predicted to increase over the next $10-15$ years. In Japan, 103,000 MPM-related mortalities are predicted to occur in the next 40 years, with the estimated peak in 2025 (1). The number of MPM-related deaths in the UK is expected to double from 1,500 to $3,000 /$ year in the 20 -year period from 2000 to 2020 (2). Although the latency from asbestos exposure is $\sim 20-30$ years (3), MPM shows rapid progression, especially in the later stages. It also has a poor prognosis, with a median overall survival period of 9-17 months, regardless of stage (4). A recent randomized study demonstrated a survival benefit of a cisplatin-pemetrexed chemotherapy regimen over cisplatin chemotherapy (5). Thus, the current key drugs for the disease are cisplatin and pemetrexed. Similar to most other solid cancers, MPM may recur due to acquired resistance to chemotherapeutic agents. The development of resistance to chemotherapeutic agents is a major impediment to the success of chemotherapy. In particular, since only a few active agents are available for MPM, resistance to these agents implies complete failure of chemotherapy.

Cisplatin binds to DNA and induces DNA cross-linking, which leads to DNA double- and single-strand breaks and causes cell death (6). Pemetrexed inhibits DNA and RNA synthesis by impairing the activity of at least 3 enzymes, thymidylate synthase (TS), glycinamide ribonucleotide formyltransferase (GARFT) and dihydrofolate reductase (DHFR), in the folate metabolic pathway, a critical pathway for purine and pyrimidine synthesis (7). The clinical effectiveness of 5-fluorouracil, another TS inhibitory agent, reportedly correlates with low TS activity in colorectal (8-11), gastric (12) and non-small cell carcinomas (13). Clinical observations in breast cancer (14) and MPM (15) suggest that pemetrexed may show similar phenomenon to 5-fluorouracil. In another study, forced high or low TS expression was linked to decreased or increased sensitivity to pemetrexed in vitro, respectively $(16,17)$. However, the precise 
interaction of pemetrexed and cisplatin in MPM remains unclear; there is no information on whether the interaction is supra-, sub- or just additive, although limited information regarding the drug-drug interactions of the 2 agents on gastric cancer (18), lung adenocarcinoma, and colorectal, breast, and ovarian cancer cell lines (19) is available. There is, however, no information on cross-resistance between the 2 agents or the precise mechanism of acquired resistance.

Therefore, elucidating the interactions and cross-resistance between cisplatin and pemetrexed, as well as the mechanism of acquired resistance, in human MPM would be crucial in improving the therapeutic strategies against the disease. In this study, the interaction of these 2 agents in 4 human MPM cell lines was assessed in vitro by performing clonogenic assays followed by isobologram analysis. To assess acquired resistance, we established cisplatin- and pemetrexed-resistant sublines of these human MPM cell lines by stepwise doseescalation of the agents. These resistant sublines were used in clonogenic assays to assess cross-resistance between the agents. In addition, expression of TS, GARFT and DHFR in these sublines and their parental cell lines were evaluated to decipher the mechanism of natural and acquired resistance.

\section{Materials and methods}

Cells and cell culture. The human mesothelioma cell lines NCI-H2452 (H2452), NCI-H28 (H28), NCI-H226 (H226) and MSTO-211H $(211 \mathrm{H})$ were obtained from American Type Culture Collection (Rockville, MD, USA). The histological type of H2452, H28 and H226 cells was epithelial, and that of $211 \mathrm{H}$ was bi-phasic. A preliminary polymerase chain reactionsingle strand conformation polymorphism analysis, including exons 5, 6, 7, 8 and 9 of TP53 of the cell lines, showed that all of them had wild-type TP53 (data not shown). These cells were cultured as a monolayer in a humidified atmosphere with $5 \%$ $\mathrm{CO}_{2}$ at $37^{\circ} \mathrm{C}$ in RPMI-1640 medium (Life Technologies, Grand Island, NY, USA), supplemented with $100 \mathrm{U} / \mathrm{ml}$ of penicillin, $100 \mathrm{mg} / \mathrm{ml}$ of streptomycin and $10 \%$ heat-inactivated fetal bovine serum.

Drugs. Cisplatin solution, at a concentration of $0.5 \mathrm{mg} / \mathrm{ml}$ (pH 2.5-5.5), was purchased from Nihon Kayaku Co. (Tokyo, Japan), and pemetrexed powder was purchased from Eli Lilly Japan K.K. (Kobe, Japan). The reagents were stored protected from light at room temperature until use. Pemetrexed was dissolved in saline at a concentration of $25 \mathrm{mg} / \mathrm{ml}(\mathrm{pH} 6.6-7.8)$ and was used within $24 \mathrm{~h}$ after preparation.

Clonogenic assay. For clonogenic assays, subconfluent cultured cells were trypsinized to obtain cell suspensions, and a varied number of cells, such that the resulting colony number per plate would be $\sim 20-50$, were immediately replated onto $6-\mathrm{cm}$ culture dishes, in triplicate, and cultured for $24 \mathrm{~h}$ in the complete medium. The agents, at various concentrations, were added to the culture medium for $24 \mathrm{~h}$ and were then replaced with new agent-free complete medium twice, followed by further incubation for 10-14 days. The obtained colonies were counted under a dissecting microscope after $1 \%$ crystal violet staining. Survival curves were drawn on the basis of the obtained data.
Isobologram analysis. Cell-survival curves with cisplatin, pemetrexed and their admixtures were analyzed by obtaining isobolograms according to the method described by Steel and Peckham (20). Briefly, mode I and II curves were drawn by calculating the additive response for the 2 agents using their individual survival curves. The mode I and II curves were assumed to represent complete independence of the agents, and exact complementation of the effect of one by the other, respectively. Isobologram analyses were carried out for 2 end-points, representing survival fractions of 0.05 and 0.02 . First, cell killing by cisplatin was read off the cisplatin-alone survival curve. Then, the pemetrexed concentration required to kill the remaining cells to reach the end-point was read off the pemetrexed-alone survival curve. This was done by starting either at $100 \%$ survival (mode I) or at the survival already reached after cisplatin pretreatment (mode II), to form the isobologram envelope. Then, the actual data from the combination of the 2 agents were plotted on the isobologram envelope. Data points in the area above, inside or below the envelope represented sub-additive, additive or supra-additive interaction of the 2 agents, respectively.

Establishment of cisplatin-resistant and pemetrexed-resistant cell lines. After subconfluent culture, cells were trypsinized and diluted with complete medium containing cisplatin or pemetrexed for $48 \mathrm{~h}$, until removal of the agents by washing twice with complete medium; this was followed by additional culture for 10-14 days in complete medium alone to obtain drug-resistant colonies large enough for cloning. The isolated colonies were mixed together for propagation to the next passage. This procedure was repeated with gradually increasing concentrations of cisplatin or pemetrexed and continued for $\sim 6$ months. The starting concentration was $0.1 \mu \mathrm{g} / \mathrm{ml}$ for both agents; the final cisplatin concentrations were 40, 10, 1 and $5 \mu \mathrm{g} / \mathrm{ml}$ for $\mathrm{H} 2452, \mathrm{H} 28, \mathrm{H} 226$ and $211 \mathrm{H}$, respectively, and the final pemetrexed concentrations were $10,000,1,10$ and $5,000 \mu \mathrm{g} / \mathrm{ml}$ for $\mathrm{H} 2452, \mathrm{H} 28, \mathrm{H} 226$ and $211 \mathrm{H}$, respectively. These resistant sublines were proven to be stably resistant even after at least 2 month-culture in drug-free complete medium. The cisplatin-resistant sublines of the 4 parental cell lines were named H2452-C, H28-C, H226-C and 211H-C, respectively. The pemetrexed-resistant sublines were similarly named H2452-P, H28-P, H226-P and 211H-P. The sensitivity of each resistant subline to cisplatin and pemetrexed was determined by performing clonogenic assays.

Quantitative reverse transcriptase-polymerase chain reaction. The expression levels of TS, GARFT and DHFR in the cells were evaluated with quantitative reverse transcriptase-polymerase chain reaction (RT-PCR). Total RNA was obtained from the cultured cells with the RiboPure ${ }^{\mathrm{TM}}$ Kit (Ambion Inc., Austin, TX, USA) according to the manufacturer's instructions. The mRNA levels were measured by TaqMan 5'-nuclease fluorogenic quantitative PCR analysis (Applied Biosystems, Foster, CA, USA) and normalized to $\beta$-actin levels according to the manufacturer's instructions. The target-specific primer sequence-sets used were 5'-CCTGAATCACATCGAGCC ACT-3' (forward) and 5'-GAAGAATCCTGAGCTTTGGG AA-3' (reverse) for TS, 5'-GGATAGTTGGTGGCAGTTCTG TT-3' (forward) and 5'-TGCATGATCCTTGTCACAAATA 

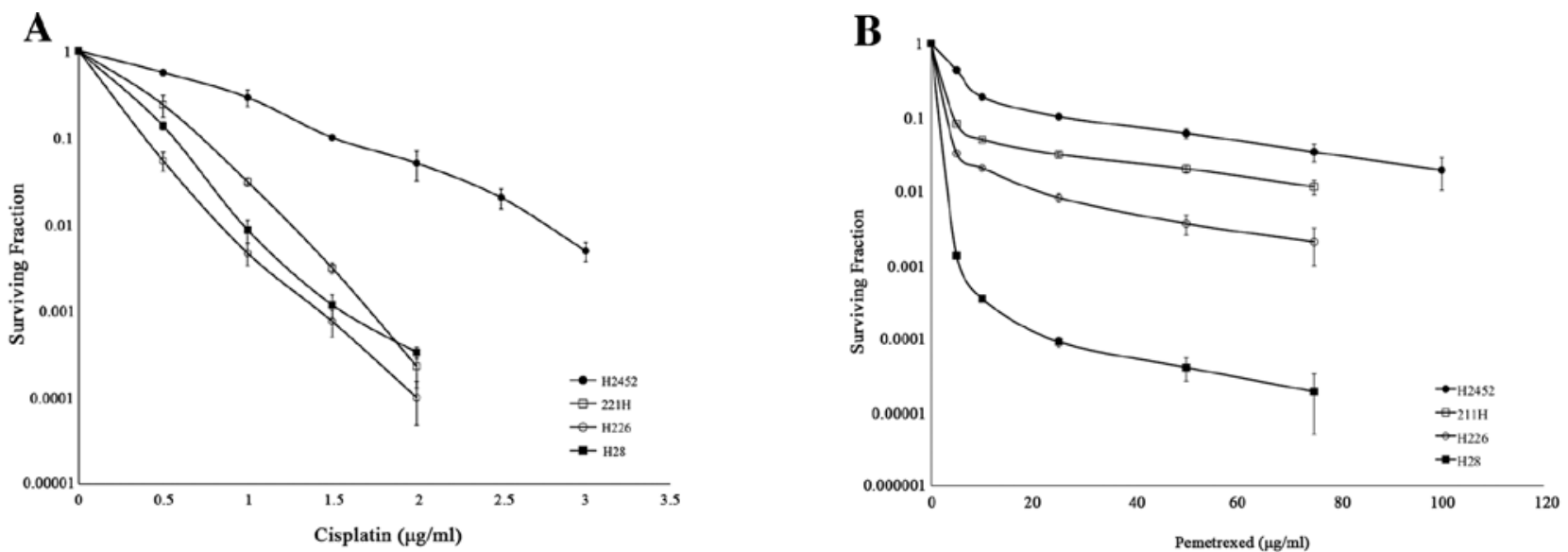

Figure 1. Survival curves for the 4 cell lines treated with cisplatin alone (A) and pemetrexed alone (B). Dots and bars represent mean and SD values, respectively. The mean \pm SD of 3 independent experiments, with each sample plated in triplicate, is shown.
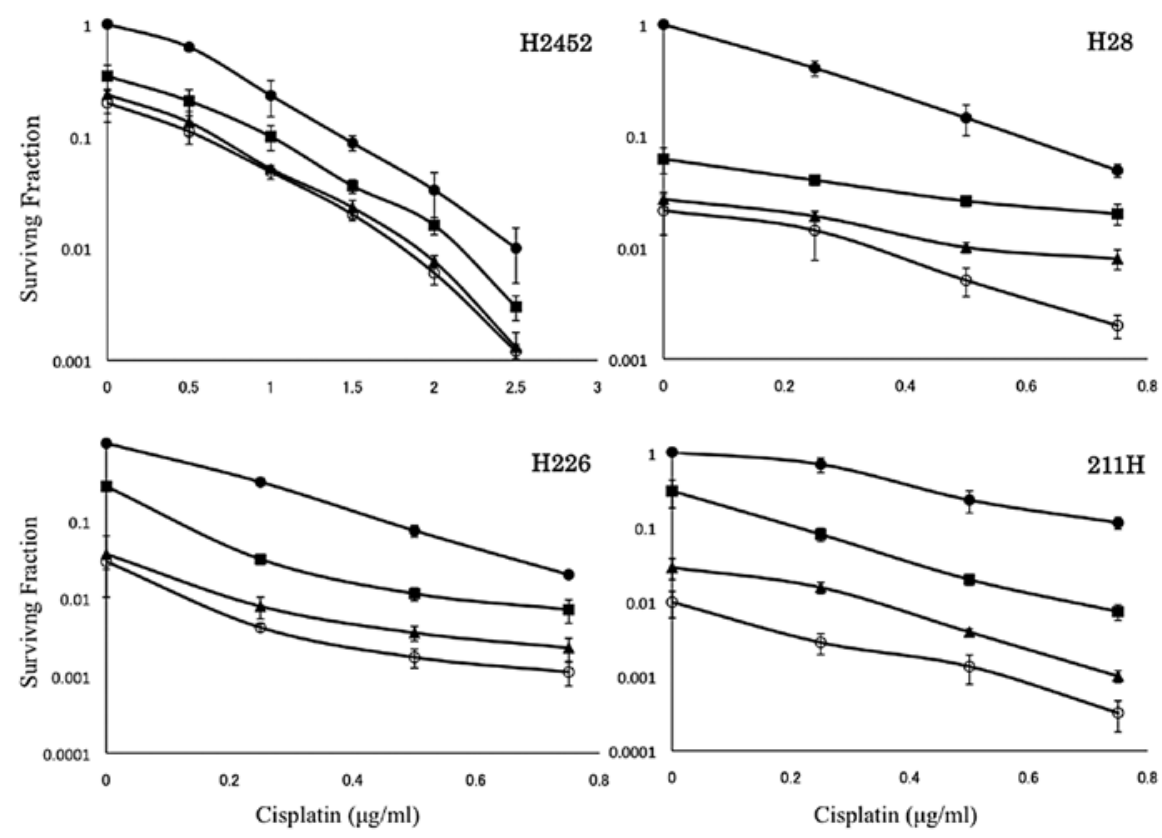

Figure 2. The survival curves for the 4 cell lines treated with a combination of cisplatin and pemetrexed at various concentrations. Concentrations for pemetrexed were 0 (closed circles), 25 (closed squares), 50 (closed triangles) and $75 \mu \mathrm{g} / \mathrm{ml}$ (open circles) in H2452; 0 (closed circles), 0.1 (closed squares), 0.15 (closed triangles) and $0.25 \mu \mathrm{g} / \mathrm{ml}$ (open circles) in $\mathrm{H} 28 ; 0$ (closed circles), 0.5 (closed squares), 1.0 (closed triangles) and $1.5 \mu \mathrm{g} / \mathrm{ml}$ (open circles) in $\mathrm{H} 226$; and 0 (closed circles), 1 (closed squares), 3 (closed triangles) and $5 \mu \mathrm{g} / \mathrm{ml}$ (open circles) in $211 \mathrm{H}$. The dots and bars represent mean and SD values, respectively. Each data point represents the mean $\pm \mathrm{SD}$ of 3 independent experiments in which each sample was plated in triplicate.

GTT-3' (reverse) for DHFR, and 5'-GCTCCCTTCTTTTAAGG GTTCAA-3' (forward) and 5'-ACCAGTAACTGTGACTCC GGT-3' (reverse) for GARFT. The probe sequences were AATTCAGCTTCAGCGAGAACCCAGACC for TS, AAG CCATGAATCACCCAGGCCATCTT for DHFR, and TGC CCATGAGCAAGCCCTGGA for GARFT (14). Quantitative RT-PCR was performed using the ABI PRISM 7000 Sequence Detection System (Applied Biosystems) with the reaction conditions consisting of reverse-transcription at $48^{\circ} \mathrm{C}$ for $30 \mathrm{~min}$ and inactivation of reverse-transcriptase at $95^{\circ} \mathrm{C}$ for $10 \mathrm{~min}$, followed by 40 cycles of amplification with denaturation at $95^{\circ} \mathrm{C}$ for $15 \mathrm{sec}$, annealing and synthesis at $60^{\circ} \mathrm{C}$ for $60 \mathrm{sec}$ in each cycle.
Statistical analysis. The mRNA levels in the drug-resistant sublines and their parental cell lines were compared and analyzed by the non-parametric Mann-Whitney U test. Differences were judged as statistically significant when p-values (two-tailed) were $<0.05$.

\section{Results}

Survival curves and isobolograms. The survival curves for the cells treated with cisplatin alone and pemetrexed alone are shown in Fig. 1A and B, respectively. Although the remaining 3 cell lines exhibited comparable sensitivity to cisplatin, $\mathrm{H} 2452$ was ostensibly more resistant to cisplatin. In addition, it 
$\mathrm{H} 2452$
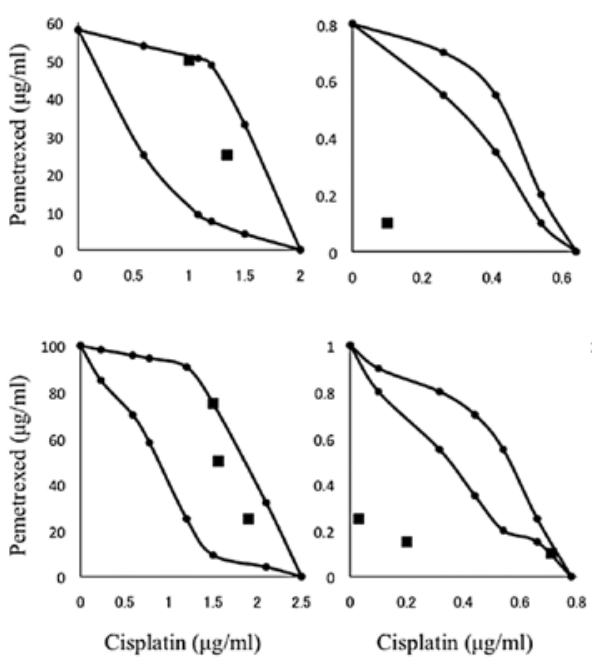

$\mathrm{H} 28$

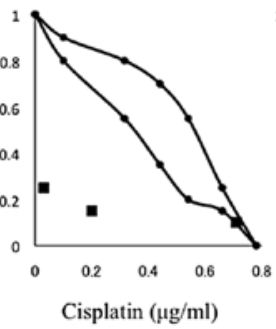

$\mathrm{H} 226$

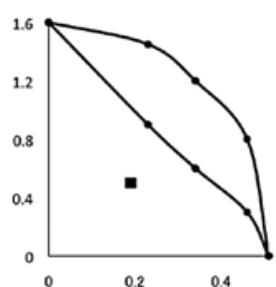

$211 \mathrm{H}$
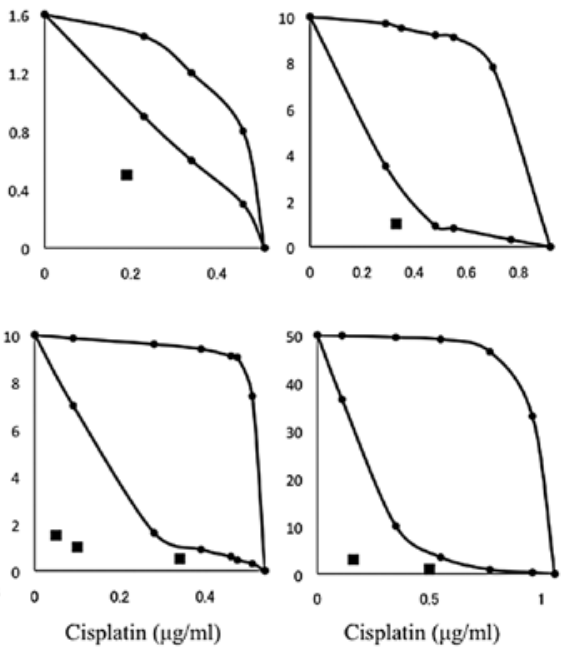

Figure 3. Isobolograms for the 4 cell lines at surviving fractions of 0.05 (top row) and 0.02 (bottom row). In each isobologram, the mode I and II curves were drawn by calculating the theoretical additive response to cisplatin and pemetrexed, resulting in an isobologram envelope. Then, the actual concentrations needed to produce the defined surviving fractions were read directly off the corresponding survival curves shown in Fig. 2 and were plotted on the charts. The interaction of the agents was additive in $\mathrm{H} 2452$ and supra-additive in the other 3 cell lines.

Table I. Summary of experimental results.

\begin{tabular}{|c|c|c|c|c|}
\hline \multirow[b]{2}{*}{ Phenotype } & \multicolumn{4}{|c|}{ Cell lines } \\
\hline & $\mathrm{H} 2542$ & $\mathrm{H} 28$ & $\mathrm{H} 226$ & $211 \mathrm{H}$ \\
\hline Baseline sensitivity to cisplatin & Resistant & Sensitive & Sensitive & Sensitive \\
\hline Baseline sensitivity to pemetrexed & Resistant & Sensitive & Intermediate & Intermediate \\
\hline Interaction of cisplatin and pemetrexed & Additive & Supra-additive & Supra-additive & Supra-additive \\
\hline Acquired cisplatin resistance & $\mathrm{x} 10$ & $\mathrm{x} 100$ & $\mathrm{x} 100$ & $\mathrm{x} 10$ \\
\hline Acquired pemetrexed resistance & $\mathrm{x} 10$ & $\mathrm{x} 100$ & $\mathrm{x} 100$ & $\mathrm{x} 10$ \\
\hline Pemetrexed to cisplatin resistance & Resistant & No & No & No \\
\hline Cisplatin to pemetrexed resistance & Sensitive & Slight resistant & Slight resistant & No \\
\hline Baseline TS expression & Intermediate & High & High & Intermediate \\
\hline TS expression in cisplatin-resistant cells & Decreased & Decreased & Decreased & NS \\
\hline TS expression in pemetrexed-resistant cells & Increased & Decreased & NS & Increased \\
\hline Baseline GARFT expression & Intermediate & Intermediate & Low & High \\
\hline GARFT expression in cisplatin-resistant cells & Decreased & Decreased & Decreased & Decreased \\
\hline GARFT expression in pemetrexed-resistant cells & Increased & Decreased & NS & Increased \\
\hline Baseline DHFR expression & Intermediate & High & Low & Low \\
\hline DHFR expression in cisplatin-resistant cells & NS & Decreased & Decreased & Decreased \\
\hline DHFR expression in pemetrexed-resistant cells & NS & Decreased & Decreased & NS \\
\hline
\end{tabular}

NS, not significant.

was also most resistant to pemetrexed. The cells were treated with combinations of cisplatin and pemetrexed at various concentrations, based on the data presented in Fig. 1, to draw the survival curves shown in Fig. 2. The isobologram envelopes for the concentration-combinations that were estimated to produce surviving fractions of 0.05 and 0.02 were drawn for each cell line, as described above. Then, the actual data of concentrations of the agents that produced the same surviving fractions were obtained from Fig. 2 and plotted on the isobologram charts. As shown clearly in Fig. 3, the interaction of cisplatin and pemetrexed was additive for $\mathrm{H} 2452$, whereas it was supra-additive or synergistic for the rest of the cell lines.

Sensitivity of the drug-resistant sublines and their crossresistance. Cisplatin-resistance ratios, determined by the $90 \%$ inhibitory concentration $\left(\mathrm{IC}_{90}\right)$ or $\mathrm{IC}_{50}$ when $\mathrm{IC}_{90}$ was not deter- 

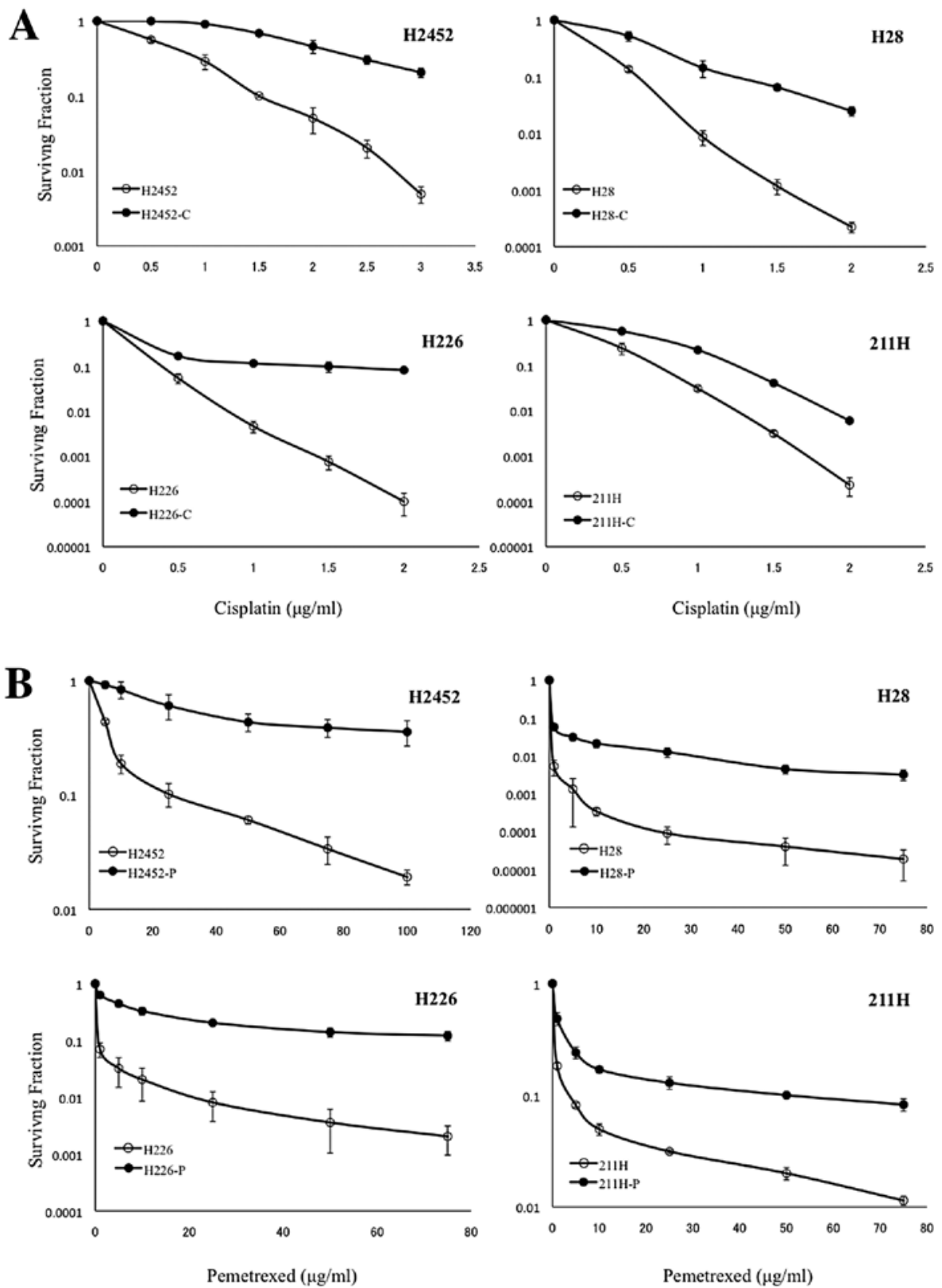

Figure 4. The survival curves for parental cell lines (open circles) and cisplatin-resistant sublines (closed circles) to cisplatin (A), and for parental cell line (open circles) and pemetrexed-resistant subline (closed circles) to pemetrexed (B) in the 4 human MPM cell lines. The dots and bars represent means and SDs, respectively. Each data point represents the mean \pm SD of 3 independent experiments in which each sample was plated in triplicate.

mined, of $\mathrm{H} 2452, \mathrm{H} 28, \mathrm{H} 226$ and $211 \mathrm{H}$ were $3.2\left(\mathrm{IC}_{50}\right.$ value of $0.60 \mu \mathrm{g} / \mathrm{ml}$ for parental line vs. $1.90 \mu \mathrm{g} / \mathrm{ml}$ for resistant cells), $2.1\left(\mathrm{IC}_{90}\right.$ value of 0.56 vs. $\left.1.20 \mu \mathrm{g} / \mathrm{ml}\right), 3.6\left(\mathrm{IC}_{90}\right.$ value of $0.39 \mathrm{vs}$. $1.42 \mu \mathrm{g} / \mathrm{ml})$ and $1.7\left(\mathrm{IC}_{90}\right.$ value of $\left.0.72 \mathrm{vs.} 1.25 \mathrm{~g} / \mathrm{ml}\right)$, respectively (Fig. 4A). Similarly, pemetrexed-resistance ratios of H2452, H28, $\mathrm{H} 226$ and $211 \mathrm{H}$ were $9.5\left(\mathrm{IC}_{50}\right.$ value of $\left.4 \mathrm{vs.} 38 \mu \mathrm{g} / \mathrm{ml}\right), 2.0\left(\mathrm{IC}_{90}\right.$ value of 0.25 vs. $0.5 \mu \mathrm{g} / \mathrm{ml}), 17.0\left(\mathrm{IC}_{90}\right.$ value of $\left.0.2 \mathrm{vs.} 3.4 \mu \mathrm{g} / \mathrm{ml}\right)$ and 14.3 ( $\mathrm{IC}_{90}$ value of $3.5 \mathrm{vs.} 50 \mu \mathrm{g} / \mathrm{ml}$ ), respectively (Fig. 4B). Cross-resistance of the 2 agents was investigated by treating cisplatin-resistant sublines with pemetrexed and vice versa, and assessing the results with clonogenic assays. Cisplatin-resistant H2452 (H2452-C) was also resistant to pemetrexed, whereas the other 3 cisplatin-resistant sublines exhibited pemetrexed sensitivities similar to those of their parental lines (Fig. 5A). In contrast, the cisplatin sensitivity in pemetrexed-resistant sublines was significantly diverse. That is, H2452-P was more sensitive, and $\mathrm{H} 28-\mathrm{P}$ and $\mathrm{H} 226-\mathrm{P}$ were less sensitive to cisplatin than their parental cells, whereas $211 \mathrm{H}-\mathrm{P}$ showed cisplatin-sensitivity indistinguishable from its parental cell line (Fig. 5B).

Expression of TS, GARFT and DHFR in drug-resistant cell lines. Relative mRNA expressions of TS, GARFT and DHFR in the cisplatin-resistant sublines were significantly lower than those in their parental cell lines, except for TS in $211 \mathrm{H}-\mathrm{C}$ and DHFR in H2452-C (Fig. 6A). In contrast, the expression levels varied widely in pemetrexed-resistant sublines relative to the expression levels in their parental cell lines. The levels of TS and GARFT in H2452-P and 211H-P were significantly higher than those in their parental cells, whereas they were significantly lower in H28-P, with those in H226-P showing 

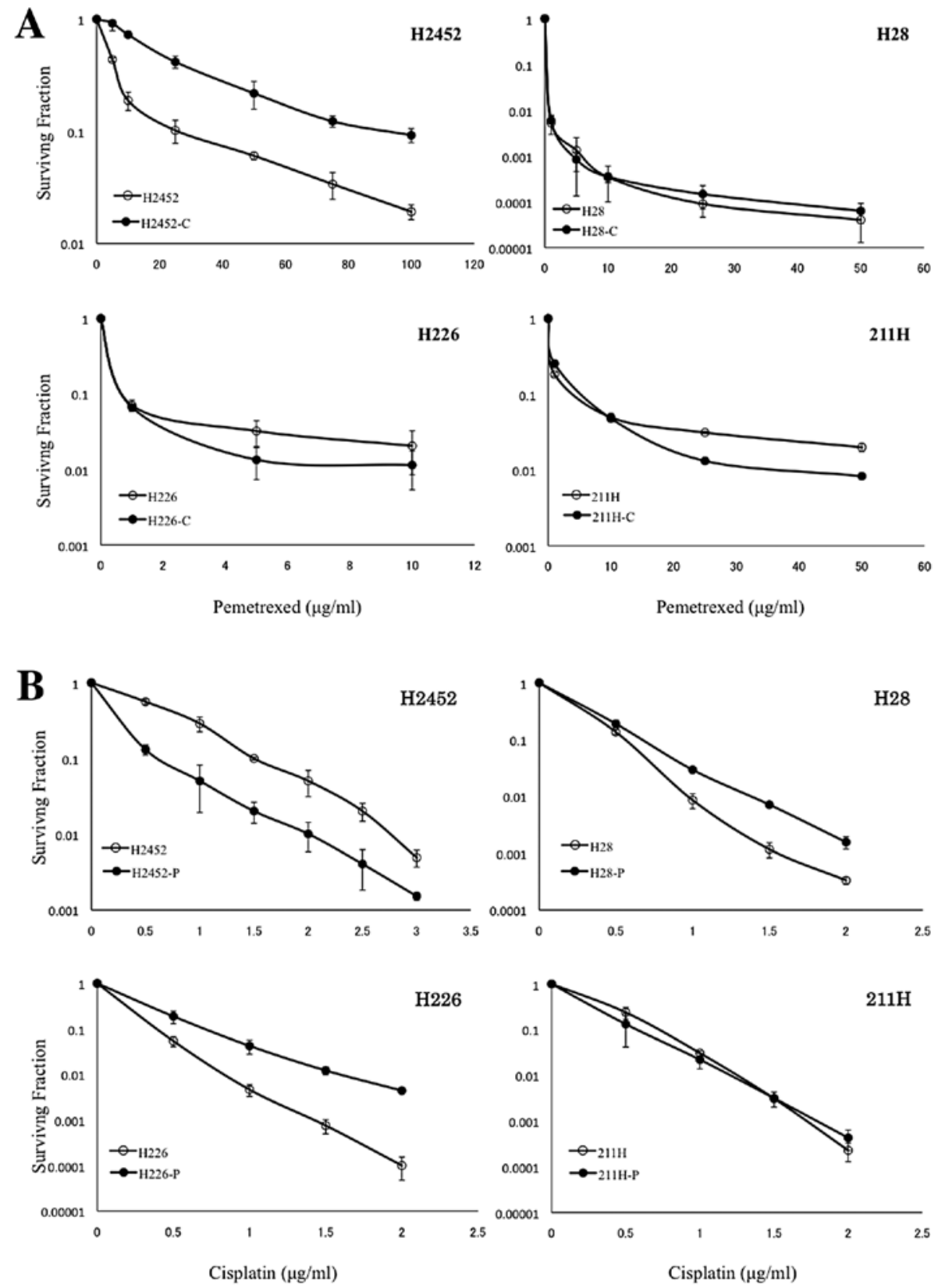

Figure 5. The survival curves for parental cell lines (open circles) and cisplatin-resistant sublines (closed circles) to pemetrexed (A), and for parental cell lines (open circles) and pemetrexed-resistant sublines (closed circles) to cisplatin (B) in the 4 cell lines for investigation of cross-resistance. H2452-C was also resistant to pemetrexed, whereas the other 3 cisplatin-resistant sublines were not resistant to pemetrexed (A). On the other hand, H2452-P was more sensitive, and $\mathrm{H} 28-\mathrm{P}$ and $\mathrm{H} 226-\mathrm{P}$ were moderately less sensitive to cisplatin than their parental cells, whereas $211 \mathrm{H}-\mathrm{P}$ exhibited similar cisplatin sensitivity as the parental cell lines (B). The dots and bars represent means and SDs, respectively. Each data point represents the mean \pm SD of 3 independent experiments in which each sample was plated in triplicate.

no significant change. The DHFR level, however, was significantly lower in H28-P and H226-P, whereas it was not altered significantly in H2452-P and 211H-P (Fig. 6B).

\section{Discussion}

In this study, baseline sensitivity to cisplatin and pemetrexed, interaction and cross-resistance of the 2 agents, and altered expression of TS, GARFT and DHFR in cisplatin- and pemetrexed-resistant sublines of 4 human MPM cell lines were investigated. Results generally varied for the 4 cell lines, indicating the heterogeneous and complex nature of MPM in terms of drug sensitivity. However, some common tendencies could be abstracted to characterize the MPM cell lines. As summarized in Table I, the interaction of cisplatin and pemetrexed was synergistic in all the cell lines except one, H2452, which was the most resistant to both the agents. Cross-resistance between the 2 agents was not observed when cisplatin-resistant cells were treated with pemetrexed, except for the cisplatin-resistant $\mathrm{H} 2452-\mathrm{C}$ subline, which was also resistant to pemetrexed. Two of the 4 pemetrexed-resistant cell lines showed mild resistance to cisplatin. The expression 
A

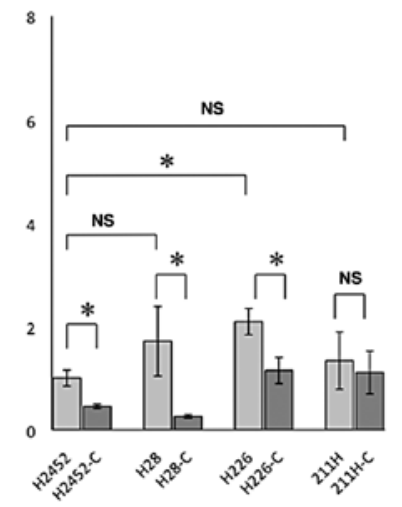

TS

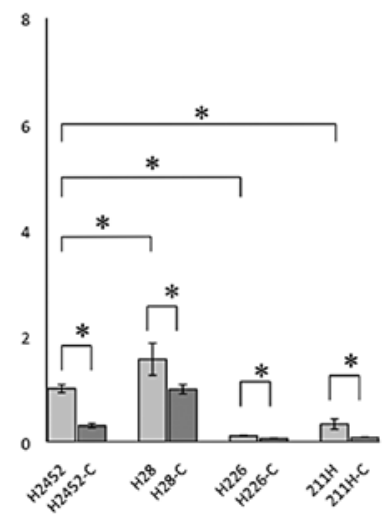

GARFT

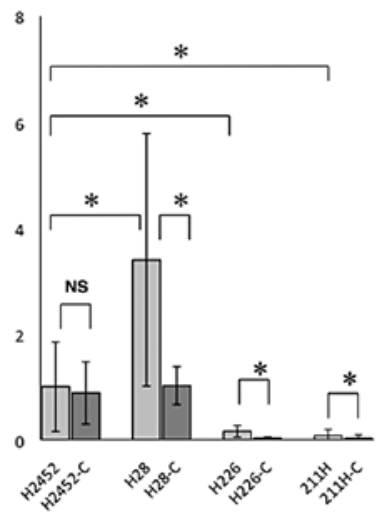

DHFR

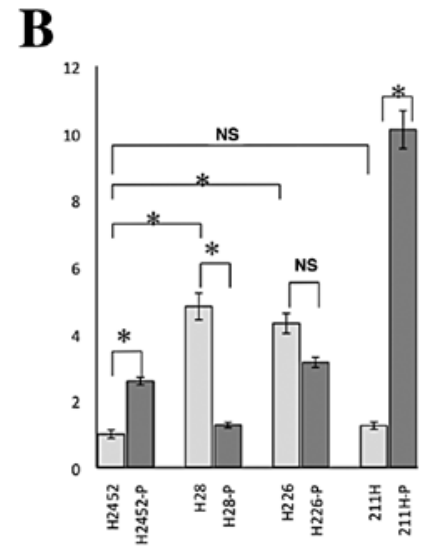

TS

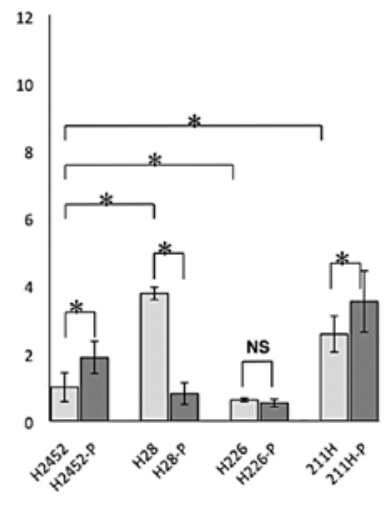

GARFT

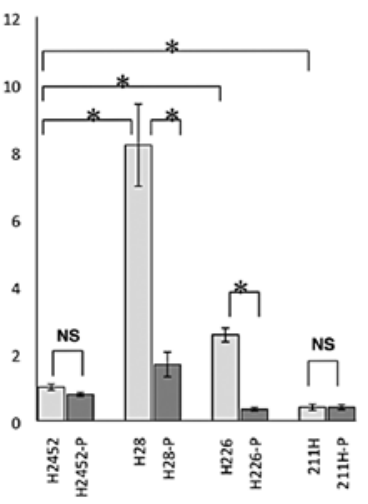

DHFR

Figure 6. Relative mRNA levels, normalized to $\beta$-actin assuming that its expression is equal in the 4 cell lines, of TS, GARFT and DHFR in cisplatin-resistant sublines (A) and in pemetrexed-resistant sublines (B), compared to their levels in parental cells. Columns and bars represent mean and SDs, respectively ( $\mathrm{n}=3$ ). An asterisk represents a statistically significant difference $(\mathrm{p}<0.05)$ and NS represents not significant.

levels of TS and GARFT were significantly elevated in only 2 of the pemetrexed-resistant sublines, suggesting that resistance mechanisms other than elevated TS expression were likely to be responsible for pemetrexed resistance in the remaining 2 cell lines.

Interaction between cisplatin and pemetrexed has been investigated in cancers other than MPM. Kim et al (18) examined 6 gastric cancer cell lines and found that the interaction was supra-additive in 2 and additive in 3 cell lines, with the remaining cell line not described. Kano et al (19) investigated the interaction of the 2 agents in 4 human cancer cell lines derived from lung adenocarcinoma and breast, ovarian, and colon cancers, and found that the interaction was scheduledependent, i.e., simultaneous administration of the 2 agents or sequential administration, with cisplatin followed by pemetrexed, resulted in an additive effect in colon carcinoma and sub-additive effects in the remaining 3 cell lines. However, sequential administration of pemetrexed followed by cisplatin resulted in a supra-additive effect in breast cancer, an additive effect in colon cancer, and a marginal effect between supraadditive and additive in the remaining 2 cell lines. On the other hand, the present study demonstrates a supra-additive effect of cisplatin and pemetrexed in 3 cell lines and an addi- tive effect in 1 human MPM cell line, in accordance with the clinical relevance of combination chemotherapy with the 2 agents for MPM. Cross-resistance between these agents was demonstrated only when 1 of the 4 cisplatin-resistant sublines was treated with pemetrexed, and 2 of the 4 pemetrexedresistant sublines were treated with cisplatin. These data differ from previous reports on the cross-resistance of these agents in non-small cell lung carcinoma. Zhang et al established 4 pemetrexed-resistant sublines from 2 non-small cell lung carcinoma cell lines, and all of them exhibited cross-resistance to cisplatin (21).

A relationship between low TS expression and sensitivity to pemetrexed has been proposed, similar to the correlation between TS expression and the clinical effectiveness of 5-fluorouracil (8-13). In a phase II study where patients with MPM were treated with pemetrexed-based chemotherapy, patients whose tumors showed low TS expression levels showed a significant survival benefit over patients with high TS expression levels (15). Although this implies that TS expression is a prognostic factor, it is not clear if TS expression in tumor tissue relates to sensitivity to the agent. Manipulating TS activity by introducing sense or antisense constructs of the TS gene resulted in reduced pemetrexed sensitivity in non-small cell 
carcinoma (16) or augmented pemetrexed sensitivity in MPM (17), respectively. Although these facts seem to provide direct evidence that TS activity is associated with pemetrexed sensitivity, they do not necessarily explain the complete mechanism of natural and acquired resistance to pemetrexed. In fact, the most naturally pemetrexed-resistant cell line, H2452, had the lowest TS expression among the 4 cell lines investigated. In addition, only 2 sublines with acquired pemetrexed resistance had TS expression levels higher than their parental cell lines. Moreover, among the pemetrexed-resistant sublines, H28-P had TS expression level significantly lower than the parental cell line. Although some clinical observations suggest a potential association between low TS expression and response to pemetrexed therapy in breast cancer (14) and MPM (15), a report on gastric cancer cell lines failed to show a correlation between TS expression and pemetrexed sensitivity (18), similar to our observations. Therefore, we assume that multiple mechanisms, including TS expression, are involved in sensitivity to pemetrexed, at least in MPM. A study of colorectal cancer cell lines demonstrated that cell lines with TP53 mutations were more resistant to antimetabolites, 5-fluorouracil, raltitrexed, and pemetrexed, than cell lines with wild-type TP53 (22). The fact that TP53 mutations are infrequent in MPM, and that all the cell lines used in this study possessed wild-type TP53, would also explain the differences in the mechanisms of pemetrexed resistance between MPM and other cancers.

Beside the information regarding the drug-drug interaction, cross-resistance and relationship between the drug-resistance and expression of the known relevant, the present research established a useful experimental model for further elucidating mechanism of sensitivity and resistance of these key drugs for MPM. Comprehensive and comparative gene expression analyses using the cell lines established here would be warranted.

In conclusion, cisplatin and pemetrexed have a synergistic effect in human MPM cells and do not show cross-resistance in general. Low TS expression does not necessarily correlate with pemetrexed sensitivity, and elevated TS expression accompanied acquired pemetrexed resistance in only 2 MPM cell lines, suggesting that multiple mechanisms may underlie natural and acquired resistance to pemetrexed in MPM. Further elucidating the underlining mechanism of the present data might uncover molecular targets to treat MPM.

\section{Acknowledgements}

This study was funded by grants from the Ministry of Education, Culture, Sports, Science and Technology of Japan.

\section{References}

1. Robinson BW and Lake RA: Advances in malignant mesothelioma. N Engl J Med 353: 1591-1603, 2005.

2. O'Brien ME: Malignant mesothelioma - the UK experience. Lung Cancer 45 (Suppl 1): S133-S135, 2004.

3. Niklinski J, Niklinska W, Chyczewska E, et al: The epidemiology of asbestos-related diseases. Lung Cancer 45 (Suppl 1): S7-S15, 2004.
4. Tsao AS, Wistuba I, Roth JA and Kindler HL: Malignant pleural mesothelioma. J Clin Oncol 27: 2081-2090, 2009.

5. Vogelzang NJ, Rusthoven JJ, Symanowski J, et al: Phase III study of pemetrexed in combination with cisplatin versus cisplatin alone in patients with malignant pleural mesothelioma. J Clin Oncol 21: 2636-2644, 2003.

6. Makimoto T, Tsuchiya S, Nakano H, et al: A phase II study of carboplatin-cisplatin-etoposide combination chemotherapy in advanced non-small cell lung cancer. Am J Clin Oncol 20: 51-54, 1997.

7. Shih C, Chen VJ, Gossett LS, et al: LY231514, a pyrrolo[2,3-d] pyrimidine-based antifolate that inhibits multiple folate-requiring enzymes. Cancer Res 57: 1116-1123, 1997.

8. Leichman CG, Lenz HJ, Leichman L, et al: Quantitation of intratumoral thymidylate synthase expression predicts for disseminated colorectal cancer response and resistance to protracted-infusion fluorouracil and weekly leucovorin. J Clin Oncol 15: 3223-3229, 1997.

9. Metzger R, Danenberg K, Leichman CG, et al: High basal level gene expression of thymidine phosphorylase (platelet-derived endothelial cell growth factor) in colorectal tumors is associated with non-response to 5-fluorouracil. Clin Cancer Res 4: 2371-2376, 1998

10. Salonga D, Danenberg KD, Johnson M, et al: Colorectal tumors responding to 5-fluorouracil have low gene expression levels of dihydropyrimidine dehydrogenase, thymidylate synthase, and thymidine phosphorylase. Clin Cancer Res 6: 1322-1327, 2000.

11. Ichikawa W, Uetake H, Shirota Y, et al: Combination of dihydropyrimidine dehydrogenase and thymidylate synthase gene expressions in primary tumors as predictive parameters for the efficacy of fluoropyrimidine-based chemotherapy for metastatic colorectal cancer. Clin Cancer Res 9: 786-791, 2003.

12. Ishikawa Y, Kubota T, Otani Y, et al: Thymidylate synthetase and dihydropyrimidine dehydrogenase levels in gastric cancer. Anticancer Res 19: 5635-5640, 1999.

13. Huang CL, Yokomise H, Kobayashi S, Fukushima M, Hitomi S and Wada $\mathrm{H}$ : Intratumoral expression of thymidylate synthase and dihydropyrimidine dehydrogenase in non-small cell lung cancer patients treated with 5-FU-based chemotherapy. Int J Oncol 17: 47-54, 2000.

14. Gomez HL, Santillana SL, Vallejos CS, et al: A phase II trial of pemetrexed in advanced breast cancer: clinical response and association with molecular target expression. Clin Cancer Res 12: 832-838, 2006.

15. Righi L, Papotti MG, Ceppi P, et al: Thymidylate synthase but not excision repair cross-complementation group 1 tumor expression predicts outcome in patients with malignant pleural mesothelioma treated with pemetrexed-based chemotherapy. J Clin Oncol 28: 1534-1539, 2010.

16. Takezawa K, Okamoto I, Okamoto W, et al: Thymidylate synthase as a determinant of pemetrexed sensitivity in non-small cell lung cancer. Br J Cancer 104: 1594-1601, 2011.

17. Flynn J, Berg RW, Wong T, et al: Therapeutic potential of antisense oligodeoxynucleotides to down-regulate thymidylate synthase in mesothelioma. Mol Cancer Ther 5: 1423-1433, 2006.

18. Kim JH, Lee KW, Jung Y, et al: Cytotoxic effects of pemetrexed in gastric cancer cells. Cancer Sci 96: 365-371, 2005.

19. Kano Y, Akutsu M, Tsunoda S, et al: Schedule-dependent interactions between pemetrexed and cisplatin in human carcinoma cell lines in vitro. Oncol Res 16: 85-95, 2006.

20. Steel GG and Peckham MJ: Exploitable mechanisms in combined radiotherapy-chemotherapy: the concept of additivity. Int J Radiat Oncol Biol Phys 5: 85-91, 1979.

21. Zhang D, Ochi N, Takigawa N, et al: Establishment of pemetrexed-resistant non-small cell lung cancer cell lines. Cancer Lett 309: 228-235, 2011.

22. Giovannetti E, Backus HH, Wouters D, et al: Changes in the status of p53 affect drug sensitivity to thymidylate synthase (TS) inhibitors by altering TS levels. Br J Cancer 96: 769-775, 2007. 